Journal of

Surgical Procedures and Case Reports

\title{
Policy towards Removal of Mini Plates in Maxillofacial Trauma -A Follow up Study of 234 Patients
}

\section{Harsha Gorrela MDS, MOMS'RCPS}

Professor and HOD, Department of Oral and Maxillofacial Surgery, MNR Dental College and Hospital, MNR Nagar, Fasalwadi, Sangareddy -502 294, Telangana, India

${ }^{\star}$ Corresponding author: Dr. Harsha Gorrela MDS, MOMS’RCPS (Glasg) Professor and HOD, Department of Oral and Maxillofacial Surgery, MNR Dental College and Hospital,MNR Nagar, Fasalwadi, Sangareddy-502 294, Telangana, India. Ph. \#91 9848389869, E-mail: dentiharsha@yahoo.com

Received Date: November 22, 2019 Accepted Date: December 14, 2019 Published Date: December 16, 2019

Citation: Harsha Gorrela (2019) Policy towards Removal of Mini Plates in Maxillofacial Trauma -A Follow up Study of 234 Patients.. J Surg Proce Case Rep 1 : 1-6.

\section{Abstract}

Aim: To analyze the incidence and factors associated with mini plates removal in maxillofacial trauma and also create a protocol for removal of miniplates in maxillofacial trauma.

Material \& Methods: Records of 234 patients attending the department of oral and maxillofacial surgery over a 8-year period were evaluated regarding the site of plating, the number of patients in whom plates were removed \& the reasons for their removal. A total of 437 stainless steel mini plates were inserted among 234 patients, among which 245 plates were inserted in mandible, 140 in the maxilla and 52 plates in a zygomatic complex region.

Results: A total of 234 patients with maxillofacial trauma were included in the study. Out of these, 172 were males and 62 were females with age range of $14-59$ yrs. A total of 437 stainless steel mini plates were inserted. A total of $48(10.98 \%)$ mini plates were removed from $28(11.96 \%)$ patients over a period of 8 years. Out of these 48 plates, $15(31.25 \%)$ plates were removed because of infection, 12 ( $25 \%$ ) plates were removed because of dehiscence, 8 ( $16.68 \%$ ) were removed because of loosened plates, $5(10.41 \%)$ were removed because of palpable plates, $5(10.41 \%)$ plates were removed upon patient request and 3 ( $6.25 \%$ ) plates were removed because of non-union.

Conclusion: Although there are conflicting opinions about the routine removal of miniplates, we follow the principle of wait and see for reasons of plate removal. The result of the present study favors our policy that is "wait and see".

Keywords: mini plates, plate removal, maxillofacial trauma.

C2019 The Authors. Published by the JScholar under the terms of the Creative Commons Attribution License http://creativecommons.org/licenses/ by/3.0/, which permits unrestricted use, provided the original author and source are credited. 


\section{Introduction}

Plates and screws for fixation of the facial skeleton have been used for more than a century. Their use started in Europe in the late 1970s and in North America in the late 1980s. Most of the available data regarding the fate of mini plates used for bony fixation has been published in the orthopedic literature. However, thousands of mini plates are being used yearly by oral and maxillofacial surgeons all over the world. Surprisingly, a review of the literature showed much philosophy and minimal data regarding the success or failure of mini plates used in the maxillofacial area. Although there is agreement that symptomatic plates should be removed, removal of asymptomatic plates from the facial skeleton is still controversial, yet which plates will become symptomatic is completely unpredictable.

Strasbourg Osteosynthesis Research Group (SORG) [1] held at the Netherlands in 1991 stated that -"A plate which is intended to assist the healing of bone becomes a non-functional implant once this role is completed. It may then be regarded as a foreign body. While there is no clear evidence to date that a plate causes actual harm, our knowledge remains incomplete. Therefore it is not possible to state with certainty that an otherwise symptomless plate left in situ is harmless. The removal of the non-functioning plate is desirable provided that the procedure does not cause undue risk to the patient".

The principal aim of this study was to establish a policy towards the removal of mini plates in maxillofacial trauma after the jaw fractures had healed.

\section{Materials \& Methods}

This Retro - Prospective study was conducted among Maxillofacial Trauma Patients who were operated in the Department of Oral and Maxillofacial Surgery between April 2005 April 2015. The entire records were revisited on or after April 2018 completing a minimum follow up of 3 years.

A total number of 279 trauma patients were treated in the Department of Oral and Maxillofacial Surgery over 7 years period. Among these, 234 patients responded for follow up, hence only 234 patients were included in the study. Among these 234 patients, 142 were fracture mandible, 55 were fracture maxilla, and 37 were zygomatic complex fractures. Among 142 mandibular fractures, 53 were parasymphysis, 38 were the angle, 19 were condyle, and 32 were body fractures.

A total of 437 stainless steel mini plates were inserted among these 234 patients, of which 245 plates were inserted in the mandible, 140 in the maxilla and 52 plates in the zygomatic complex region [Figure. 1]. A proforma was made for all the patients, data were tabulated and analyzed.

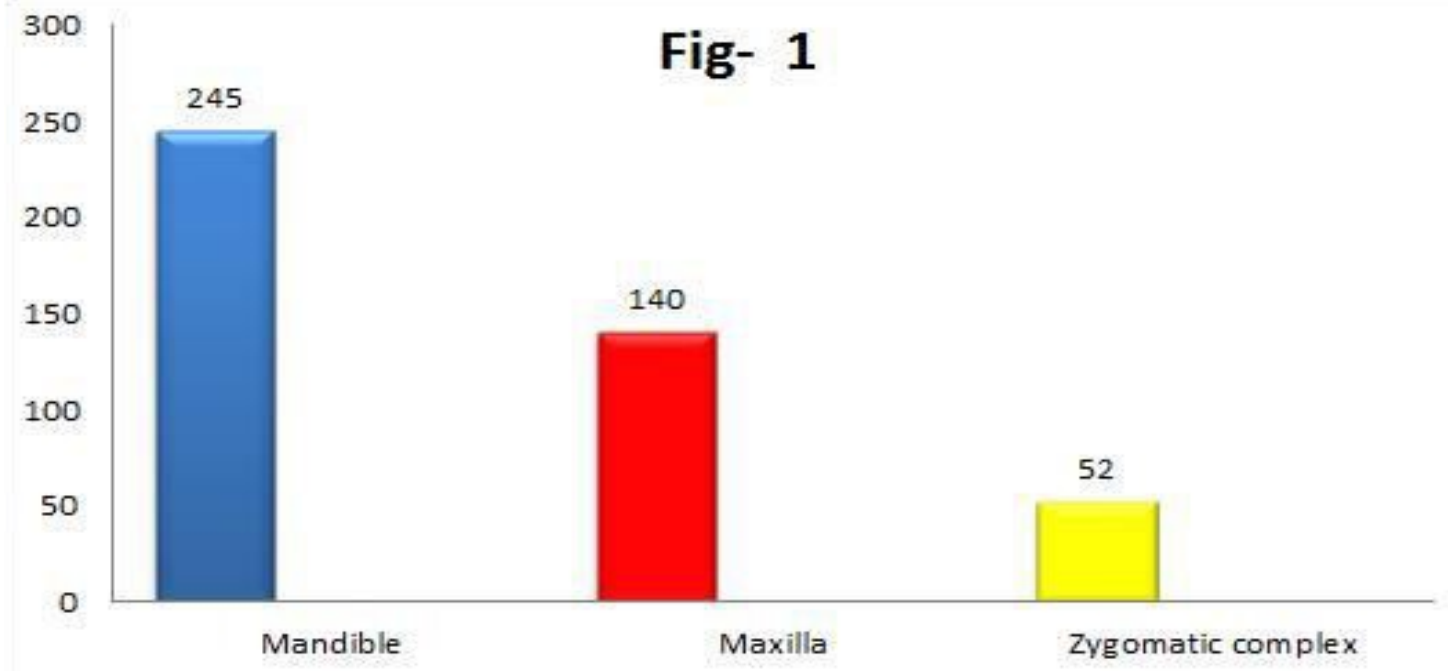

Number of Mini Plates Inserted 


\section{Results}

A total of 234 patients with maxillofacial trauma were included in the study. Out of these, 172 were males and 62 were females with an age range of $14-59$ yrs. A total of 437 stainless steel mini plates were inserted, of which 48 (10.98\%) mini plates were removed from $28(11.96 \%)$ patients over a period of 7 years.

Out of 437 mini plates inserted, 245 (56.06 \%) plates were inserted in mandible, 140 (32.04\%) plates in maxilla, 52 $(11.9 \%)$ plates in zygomatic complex region. In the mandible alone 126 (51.4\%) mini plates were inserted in the parasymphysis region, $52(21.22 \%)$ plates in angle region, 25 (10.2\%) plates in condyle and $42(17.14 \%)$ plates in body region were inserted. Out of 48 mini plates removed , 12 (25\%) mini plates were removed from parasymphysis region, 12 ( $25 \%$ ) plates were removed from angle region, 9 (18.75\%) plates were removed from body region, 9 (18.75\%) plates were removed from zygomatic complex region and $6(12.5 \%)$ mini plates were removed from maxillary region [Figure.2].

Out of 126 mini plates inserted in the parasymphysis region, 12 (9.52\%) plates were removed from parasymphysis region. Out of 52 mini plates inserted in the angle region, 12 (23.26\%) plates were removed from the angle of the mandible. Out of 42 mini plates inserted in the body region, 9 (21.42\%) plates were removed from the body of the mandible. Out of 52 mini plates inserted in a zygomatic complex region, 9 (17.3\%) plates were removed from the zygomatic complex region. Out of 140 mini plates inserted in the maxilla, $6(4.28 \%)$ plates were removed from maxilla [Figure.3].

Out of the 48 mini plates removed, 15 (31.25\%) plates were removed because of infection, 12 (25\%) plates were removed because of dehiscence, 8 (16.68 \%) were removed because of loosened plates, 5 (10.41\%) were removed because of palpable plates, 5 (10.41\%) were removed upon patient request and 3 $(6.25 \%)$ were removed because of non-union [Figure 4 ].

Taking into account, the time elapsed before removal of mini plates, 27 (56.25\%) mini plates were removed within 8 weeks of insertion because of plate related symptoms such as infection -15 ( $31.25 \%$ ) and dehiscence -12 ( $25 \%)$. Rest 21 (43.75 $\%)$ plates were removed because of $8(16.68 \%)$ loosened plates within 8 months, 5 ( $10.41 \%)$ palpable plates within 7 months, $5(10.41 \%)$ plates were removed upon patient request within 2 years \& 3 (6.25\%) plates were removed due to non- union within 3 months. Patient's request was because of concern about the permanent implant and also plate being palpable. Factors contributing to non-union were a poor medical condition. None of the patients neither requested nor showed any concern for plate removal after 2 years of insertion.

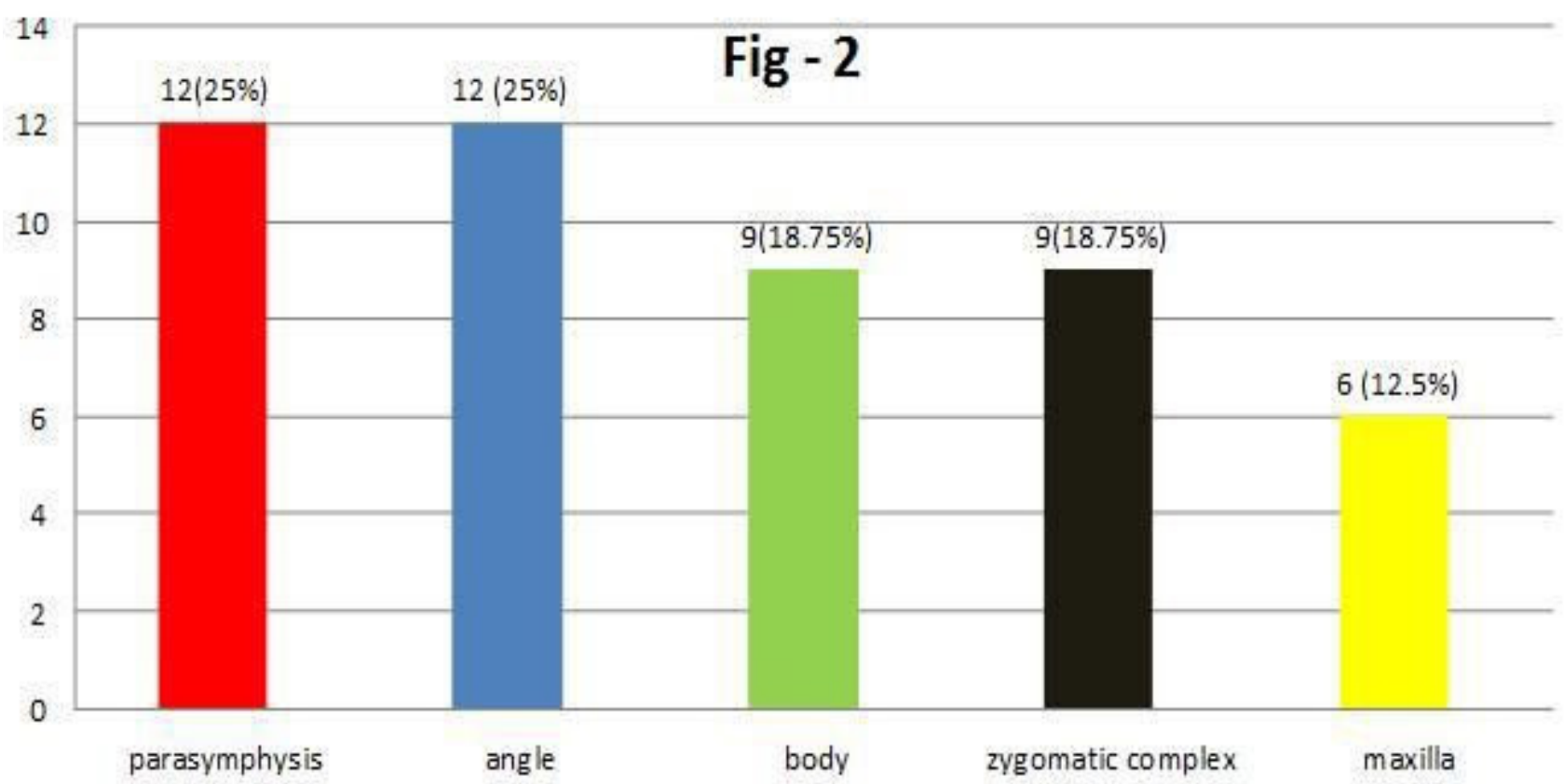

Number of Miniplates removed 


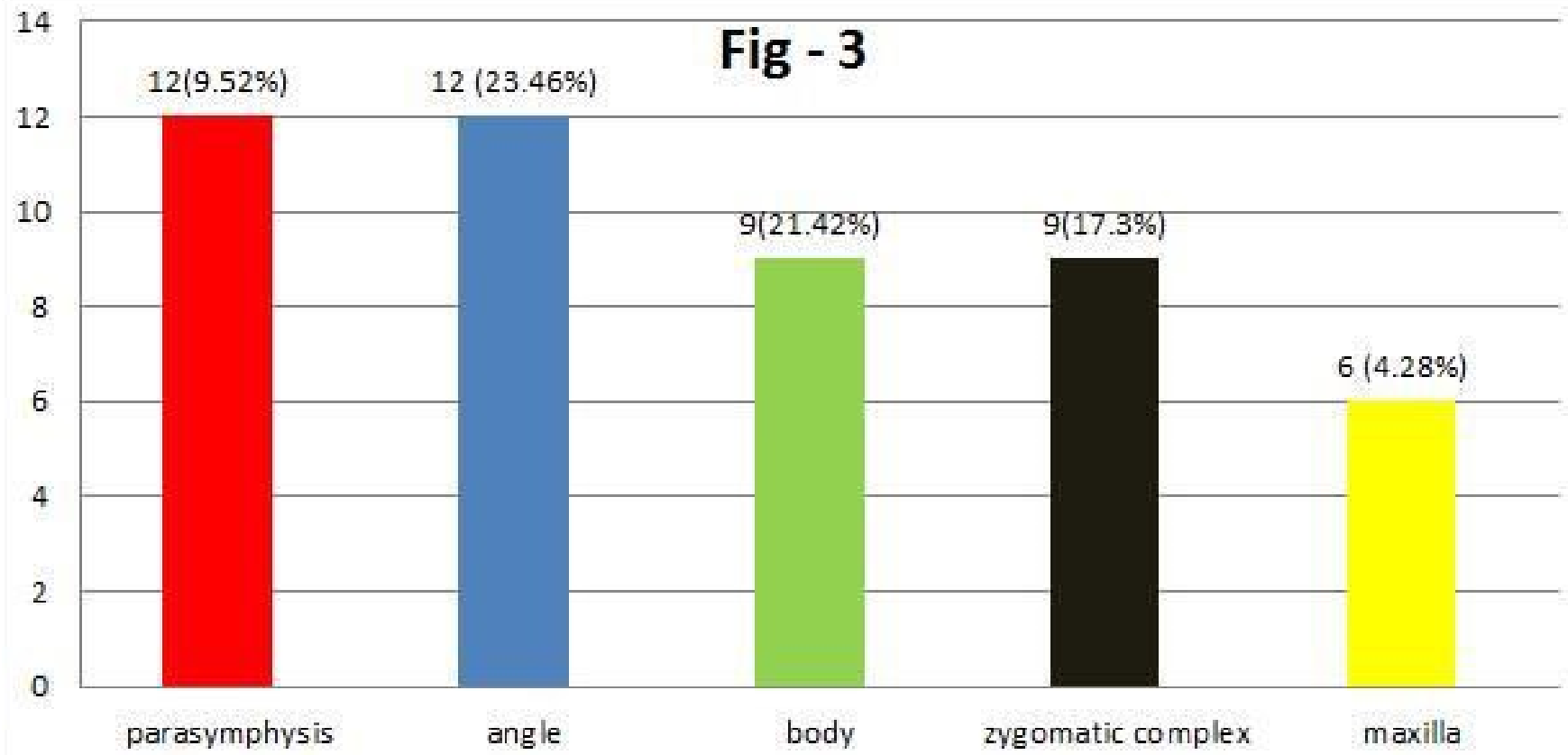

Site wise miniplates removal

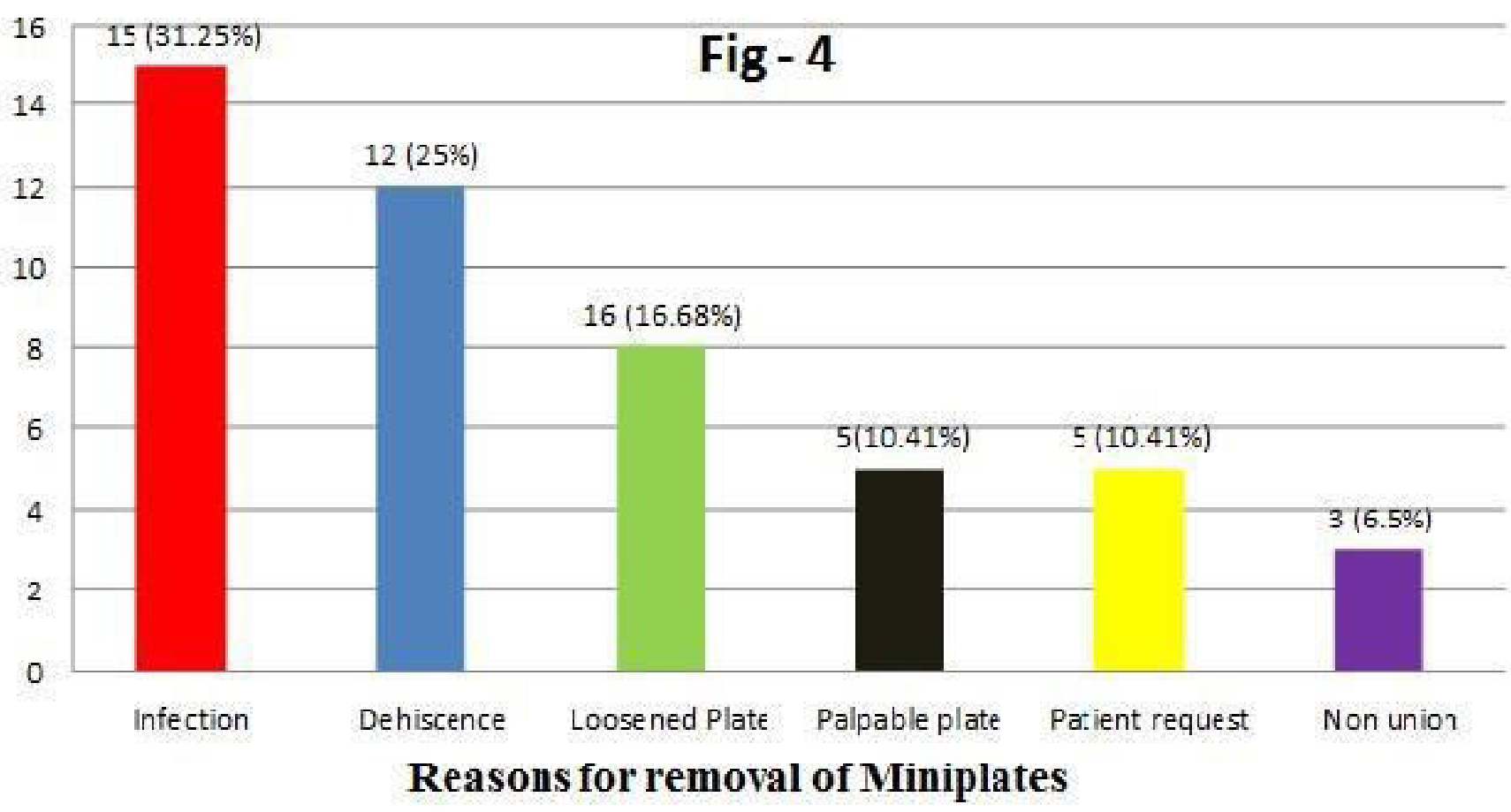




\section{Discussion}

In our 7 years follow up study of 234 patients who underwent internal fixation with 437 stainless steel mini plates, around 48 (10.98\%) plates were removed from 28 (11.96\%) patients which correlate with the study of Rallis G et al [2]. Out of these, 27 ( $56.25 \%$ ) were purely due to plate related symptoms such as infection [15 plates( $31.25 \%)$ ] and dehiscence [12 plates (25\%)] which correlates with the studies of Rallis G et al [2], Thoren H [3], Kuhlefelt $\mathrm{M}$ et al [4], Francel et al [5].

Loosened plates 8 (16.68 \%) was the other reason for removal of plates which could be due to local effect of cytokines or proteolytic enzymes that are capable of inducing bone resorption and are produced by activated macrophages after exposure to particulate metal debris at bone-implant interface as told by Al saffar et al [6]. Thermal damage may occur even during careful drilling of the screw hole and may eventually lead to screw loosening by aseptic necrosis as told by Millar BG et al [7]. 5 (10.41\%) mini plates were removed due to palpable plates. 5 $(10.41 \%)$ plates were removed upon the patient's request because of the concern of permanent implant. 3 (6.25\%) more plates were removed because of nonunion due to a poor medical condition. The location of plates on the facial skeleton may influence symptoms and hardware removal. In our study, most of the mini plates were removed from parasymphysis region 12( $25 \%$ ) and angle 12 ( $25 \%$ ) region, followed by body 9 ( $18.75 \%$ ) , zygomatic complex 9 ( $18.75 \%$ ) and maxilla 6 ( $12.5 \%$ ) region. Our findings concur with those of Brown et al [8], Mosbah et al [9] and Rallis G et al [2] and differ from those of Francel et al $[5]$.

Considering the time the plates remained in situ before removal, most of the plates 27 (56.25\%) were removed within 2 months. The rest of the plates $21(43.75 \%)$ were removed within 24 months. No plate that remained in situ for more than 24 months required any plate removal which correlates with the study of Brown et al [8], Kuhlefelt M et al [4].

In the literature, there are many studies that favor routine removal of mini plates. Cawood recommended routine removal of stainless steel mini plates after 3 months to avoid plate interfering with jaw function. Iizuka and Lindqvist [11] routinely removed stainless steel plates about a year postoperatively because there were no grounds for leaving a foreign metal object in situ after the bone had healed. Moberg et al [12] advised routine removal of nickel chromium and cobalt chromium implants af- ter satisfactory healing because metal elements released from the surface could induce allergic sensitization.

Similarly, there are many studies that advice against routine removal of mini plates. Rosenberg et al [13] removed titanium miniplates only if the patient had symptoms or if they become infected or undergo wound breakdown. Brown et al [8] in retrospective study of 279 miniplates used as permanent implants challenged the practice of routine removal of stainless steel miniplates 3 or 4 months after insertion.

Strasbourg Osteosynthesis Research Group (SORG) [1] held at the Netherlands in 1991 stated that - "A plate which is intended to assist the healing of bone becomes a non-functional implant once this role is completed. It may then be regarded as a foreign body. While there is no clear evidence to date that a plate causes actual harm, our knowledge still remains incomplete. Therefore it is not possible to state with certainty that an otherwise symptomless plate left in situ is harmless. The removal of the non-functioning plate is desirable provided that the procedure does not cause undue risk to the patient".

Considering the above findings, we prefer to follow the following protocol for the removal of Mini plates. Removal of Mini plates in our Maxillofacial unit is done only when there is

1. Presence of Plate related symptoms

2. Patient request or concern

We prefer to wait and see for a cause for removal rather than routine removal of plates after a particular time period.

\section{Conclusion}

The present study concluded that the main reasons for mini plate removal after maxillofacial trauma in our unit were 1. Infection ( $31.25 \%$ ) accounted for the maximum number of plate removal

2. Dehiscence (25\%) leading to plate exposure

3. Loosened plates (16.68\%)

4. Palpable plates (10.41\%).

5. Patient request $(10.41 \%)$ because of the concern for permanent implant

6. Nonunion $(6.25 \%)$.

Although there are conflicting opinions about routine mini plates removal, we follow the principle of wait and see for reasons of plate removal. The result of the present study favors 
our policy that is "wait and see". We also further conclude that it is wise to leave a non-functioning symptomless miniplates insitu.

\section{References}

1. (1991) Strasbourg Osteosynthesis Research Group (SORG) recommendations.

2. Rallis G, Mourouzis C, Papakosta V, Papanastasiou G, Zachariades N (2006) Reasons for miniplate removal following maxillofacial trauma: a 4-year study. J Craniomaxillofac Surg 34: 435-439.

3.

Thorén H, Snäll J, Hallermann W, Kormi E, Törnwall J (2008) Policy of routine titanium miniplate removal after maxillofacial trauma. J Oral Maxillofac Surg 66: 1901-1904. 4. Kuhlefelt M, Laine P, Suominen-Taipale L, Ingman T, Lindqvist C, et al. (2010) Risk factors contributing to symptomatic miniplate removal: a retrospective study of 153 bilateral sagittal split osteotomy patients. Int J Oral Maxillofac Surg 39: 430-435.

5. Francel TJ, Birely BC, Ringelmann PR, et al. (1992) The fate of plates and screws after facial fracture reconstruction. Plast Reconstr Surg 90: 568.

Submit your manuscript to a JScholar journal and benefit from:

ब Convenient online submission

ๆ Rigorous peer review

I Immediate publication on acceptance

I Open access: articles freely available online

q High visibility within the field

- Better discount for your subsequent articles

Submit your manuscript at http://www.jscholaronline.org/submit-manuscript.php 